\title{
CANONICAL TRANSFORMATIONS AND GRAPH THEORY मे
}

\author{
Chjan C. LIM \\ Mathematics Department, University of Michigan, Ann Arbor, MI 48109, USA \\ and Institute for Mathematics and its Applications, Minneapolis, MN 55455, USA
}

Received 29 January 1989; accepted for publication 10 April 1989

Communicated by D.D. Holm

\begin{abstract}
In the class of symplectic matrices, two infinite subsets are generated by binary spanning trees. For given $N$, the procedure begins with the complete graph, $\mathrm{K}_{N}$ to which $N-1$ virtual vertices are added in well-defined ways. Then spanning binary trees that connect the $2 N-1$ vertices are obtained and from these trees, explicit formulae give the symplectic matrices. These matrices define linear canonical transformations for $N$-body problems in vortex dynamics, plasma physics as well as celestial mechanics.
\end{abstract}

\section{Introduction}

In the past, there have been many significant applications of graph theory in theoretical physics. Outstanding amongst these are the Feynman diagrams in quantum field theory [1] and Mayer's cluster expansion in statistical mechanics [2]. The results in this note represent a new application of graph theoretical ideas to canonical transformation for classical Hamiltonian systems. More specifically, the transformations discussed here are applicable to a variety of $N$-body problems in vortex dynamics [3], plasma physics [4] and celestial mechanics [5]. In gravitational $N$-body problems, isolated cases of these canonical transformations have received the attention of astrophysicists and mathematicians who know them as the Jacobi coordinates [5]. One of the aims here is to give a unified treatment of these transitions in terms of spanning binary trees, that generalizes the Jacobi coordinates. A $2 N \times 2 N$ matrix $M$ is said to be symplectic if

$$
\mathbf{M}^{\mathrm{t}} \mathrm{JM}=\mathbf{J}, \quad \mathrm{J}=\left[\begin{array}{cc}
0 & \mathrm{I}_{N} \\
-\mathrm{I}_{N} & 0
\end{array}\right] \text {. }
$$

In the context of Hamiltonian mechanics, a transformation in phase space, $T^{*}\left(\mathbf{R}^{N}\right)$,

$\boldsymbol{Q}=\boldsymbol{Q}(\boldsymbol{q}, \boldsymbol{p}), \quad \boldsymbol{P}=\boldsymbol{P}(\boldsymbol{q}, \boldsymbol{p})$,

is canonical if its Jacobian matrix is symplectic. From (2), it is clear that the Jacobian matrix $M$ has the form

$\mathbf{M}=\left[\begin{array}{ll}\mathbf{A} & \mathbf{B} \\ \mathbf{C} & \mathbf{D}\end{array}\right]$,

where A, B, C, D are $N \times N$ matrices. Applying (1)-(3) it is clear that the following conditions are equivalent to the above definition of a symplectic matrix:

$\mathbf{A}^{\prime} \mathbf{C}, \mathbf{B}^{\prime} \mathbf{D}$ are symmetric $N \times N$ matrices,

$A^{\prime} D-C^{\prime} B=I_{N}$.

The results in this paper have been presented in seminars at Brown University and the University of Minnesota. 
Also evident is the observation that these conditions for a canonical transformation are independent of the Hamiltonian function.

In this note, we consider two classes of linear canonical transformations; equivalently we discuss a graphtheoretic method for generating symplectic matrices. These matrices fall into two general classes; they take the following forms:

$\mathbf{M}_{1}\left[\begin{array}{ll}\mathbf{A} & 0 \\ 0 & \mathbf{A}\end{array}\right], \quad \mathbf{A}$ is an $N \times N$ real matrix

and

$\mathbf{M}_{2}=\left[\begin{array}{ll}\mathbf{A} & 0 \\ 0 & \mathbf{D}\end{array}\right], \quad \mathbf{D} \neq \mathbf{A}$ are $3 N \times 3 N$ real matrices.

In the first case, $(4 a)$ is satisfied (trivially) while $(4 b)$ translates into

$\mathbf{A}^{\prime} \mathbf{A}=\mathbf{I}_{N}$.

In the other case, $(4 a)$ is also trivial and $(4 b)$ becomes

$A^{\prime} D=I_{3 N}$.

Although these symplectic matrices are identified with canonical transformations independent of the particular Hamiltonian function, to each class of matrices belongs a natural family of Hamiltonians. To distinguish them, we will call those associated with $\mathbf{M}_{1}$ first-order Hamiltonians, and those associated with $\mathbf{M}_{2}$ secondorder Hamiltonians. It turns out that these labels are appropriate in a different sense. The second-order Hamiltonians arise naturally in the $N$-body problems of celestial mechanics (cf. refs. [5-7]). They involve Newton's second law of motion directly and their Hamilton's equations are obtained from the second-order equations of motion (second-order time derivatives) after the usual reduction [5]. On the other hand, the first-order Hamiltonians, such as those from vortex dynamics [3], and plasma physics [4], do not involve accelerations and forces; their Hamilton's equations are not derived from equations of motion with second-order time derivatives.

We will describe these Hamiltonians explicitly in section 2. In section 3, we show how a complete graph $\mathrm{K}_{N}$ can be associated with these Hamiltonians. Next we will describe a procedure for generating spanning tree graphs, starting from $\mathrm{K}_{N}$. Finally, explicit formulae for the symplectic matrices will be given in terms of the spanning tree graphs. Instead of complete proofs which are published elsewhere [8,9], we give some illuminating examples of our procedure in section 4 of this note. For graph-theoretical concepts (which are essentially selfevident ), we refer the reader to refs. $[10,11]$.

\section{Hamiltonians}

The first-order Hamiltonians described in section 1 have the general form

$H(Z)=\operatorname{Re}\left\{\sum_{j \neq k=1}^{N} \Gamma_{j} \Gamma_{k} F_{j k}\left(Z_{j}-Z_{k}\right)\right\}, \quad Z=\left(Z_{1}, \ldots, Z_{N}\right)$,

where $Z_{j} \in \mathbb{C}$ give the positions of $N$ particles in the plane, and $\Gamma_{j} \in \mathbb{R}$ are their "weights". $F_{j k}$ are analytic functions of one complex variable (with the possibility of isolated singularities). The conjugate variables (canonical coordinates) are a1 $^{2}$

"1 In this paper, we will focus on "weights" of one sign; the general case is only technically different. 
$q_{j}=\sqrt{\left|\Gamma_{j}\right|} x_{j}, \quad p_{j}=\sqrt{\left|\Gamma_{j}\right|} y_{j}, \quad Z_{j}=x_{j}+\mathrm{i} y_{j}$.

A concrete example from vortex dynamics [4] is given by

$F_{j k}=$ logarithm

in which the particular branch of the complex logarithm function is immaterial because the Hamiltonian involves only the real part.

The second-order Hamiltonians are the usual $N$-body Hamiltonians with general potential functions,

$H(\boldsymbol{q}, \boldsymbol{p})=\sum_{j=1}^{N} \frac{\left|p_{j}\right|^{2}}{2 m_{j}}-U(\boldsymbol{q})$.

where the Euclidean norm in $\mathbb{R}^{3}$ is

$|\boldsymbol{p}|^{2}=\left(p_{j}^{(1)}\right)^{2}+\left(p_{j}^{(2)}\right)^{2}+\left(p_{j}^{(3)}\right)^{2}$

and the potential has the form

$U(\boldsymbol{q})=G \sum_{j \neq k}^{N} \frac{m_{j} m_{k}}{\left|q_{j}-q_{k}\right|^{\prime}}$.

$G$ is a real constant, $m_{j}$ are the masses of the particles and

$\left|q_{j}-q_{k}\right|^{\prime} \equiv\left[\left(q_{j}^{(1)}-q_{k}^{(1)}\right)^{2}+\left(q_{j}^{(2)}-q_{k}^{(2)}\right)^{2}+\left(q_{j}^{(3)}-q_{k}^{(3)}\right)^{2}\right]^{1 / 2}$,

$l$ is a real number greater than zero, and the Newtonian case is obtained when $l=1$.

\section{Spanning binary trees and symplectic matrices}

Although the symplectic matrices we are concerned with can be discussed without reference to the Hamiltonians in section 2, we want to motivate the introduction of graph-theoretic concepts by starting our discussion with $\mathrm{N}$-body problems with pair-wise (2-body) interactions. Both classes of Hamiltonians in section 2 are of this type.

If we connect all possible pairs of points in the plane given by $Z=\left(Z_{1}, \ldots, Z_{N}\right)$ in (9), the resulting picture is the complete graph, $\mathbf{K}_{N}$. Similarly, for the Hamiltonian (12), we obtain $\mathbf{K}_{N}$ where the vertices are located in $\mathbb{R}^{3}$. Thus, we are justified in associating a complete graph $\mathrm{K}_{N}$ with the abstract $N$-body problem with pairwise interactions. Furthermore, each particle (vertex) has a "weight"; in (9) they are $\Gamma_{j}$ (which represents vorticity in vortex dynamics, (11)).

Our procedure consists of three parts: (a) an averaging step that defines a binary operation INTER, (b) based on INTER, $N-1$ virtual vertices are added to the original $N$ vertices in $\mathrm{K}_{N}$ to make a spanning tree with $2 N-1$ vertices $G_{k}^{s}(N)$, and (c) for each virtual vertex in the tree, eorresponding rows in the matrices $A$ and D (5), (6) are given explicitly by well-defined rules.

The binary operation, INTER is given by

$\mathrm{V}=\operatorname{INTER}(\mathrm{A}, \mathrm{B})=\frac{\Gamma(\mathrm{A}) \mathrm{A}+\Gamma(\mathrm{B}) \mathrm{B}}{\Gamma(\mathrm{A})+\Gamma(\mathrm{B})}, \quad \Gamma(\mathrm{A}), \Gamma(\mathrm{B}) \in \mathbb{R} ; \quad \mathrm{V}, \mathrm{A}, \mathrm{B} \in \mathbb{C}$,

where $\mathrm{V}=\mathrm{V}_{j}$ is a new virtual vertex and the arguments $\mathrm{A}, \mathrm{B}$ can be either a previously generated virtual vertex, e.g. $\mathrm{V}_{h}$ for some $h<j$ or one of the original vertices in $\mathrm{K}_{N}$, e.g. $Z_{q}, q=1, \ldots, N$. To complete step (a), the weight of the new virtual vertex is calculated as follows:

$\Gamma(\mathrm{V})=\Gamma(\mathrm{A})+\Gamma(\mathrm{B})$. 
This operation is governed by the rules

(i) vertices, virtual or original can only be used once as arguments in INTER;

(ii) to begin, $k \leqslant[N / 2]$ disjoint edges in $\mathrm{K}_{N}$ are pre-selected to produce the first $k$ virtual vertices, $\mathrm{V}_{j}$ where $1 \leqslant j \leqslant k$;

(iii) the process stops when there are no eligible pairs of vertices remaining that can be used as arguments of INTER.

The original vertices, $Z_{q}$, are said to be in tier 0 , while the first $k$ virtual vertices in (ii) are said to be in tier 1 and virtual vertices generated by subsequent applications of INTER are said to be in tiers $\geqslant 2$, according to the number of times INTER has been used.

After a relabelling (by permutations on $N$ integers) of the tier- 0 vertices, the tier- 1 virtual vertices can without loss of generality be taken to be

$\mathrm{V}_{j}=\operatorname{INTER}\left(Z_{2 j-1}, Z_{2 j}\right), \quad j=1, \ldots, k$.

The number of tier-1 vertices is assigned to the index $k$ in the labels $\mathrm{G}_{k}^{s}(N)$ for the spanning trees with $2 N-1$ vertices and $\mathrm{A}_{N}^{k}$, the associated tree which will be introduced next.

In order to organize our procedure, we introduce an associated tree $\mathrm{A}_{N}^{k}$ for each $N$ and each $k \leqslant[N / 2]$. The branches of $\mathrm{A}_{N}^{k}$ code the different routes for constructing $\mathrm{G}_{k}^{s}(N)$ from $\mathrm{K}_{N}$. For given $N$ and $k \leqslant[N / 2]$, the associated tree, $A_{N}^{k}$, is constructed according to the rules

(i) the root is always labelled $V_{1}$,

(ii) the branches code the generation of virtual vertices in tiers $\geqslant 2$; beginning with $V_{k+1}$ and ending with $\mathrm{V}_{N-1}$, the vertices of $\mathrm{A}_{N}^{k}$ below the root appear in the same order (increasing subscript) on each branch; it is assumed that the tier-1 vertices $\mathrm{V}_{j}$ for $j=1, \ldots, k$ are generated as in (15),

(iii) the edges of $\mathrm{A}_{N}^{k}$ are labelled by boxed vertices consisting of only the $\mathrm{Z}_{q}, 2 k+1 \leqslant q \leqslant N$, and the tier-1 vertices, $\mathrm{V}_{j}, j=1, \ldots, k$,

(iv) an edge, $\nabla=(u, \omega)$ where $u$ is the upper vertex encodes the operation $\omega=\operatorname{INTER}(u, v)$.

We now give an example of the above step for generating spanning trees, which is part (b) of the whole procedure. The example chosen is $\mathrm{A}_{5}^{2}$ (cf. fig. 1) where $k=2$ is the maximum number of tier-1 virtual vertices or equivalently, disjoint edges in the complete graph, $K_{5}$. We begin by generating the tier-1 vertices

$\mathrm{V}_{1}=\operatorname{INTER}\left(Z_{1}, Z_{2}\right), \quad \mathrm{V}_{2}=\operatorname{INTER}\left(Z_{3}, Z_{4}\right)$.

The root of $A_{5}^{2}$ is denoted by $V_{1}$. From the root there are two distinct ways to generate the tier- 2 vertex, $V_{3}$. On the right branch (fig. 1),

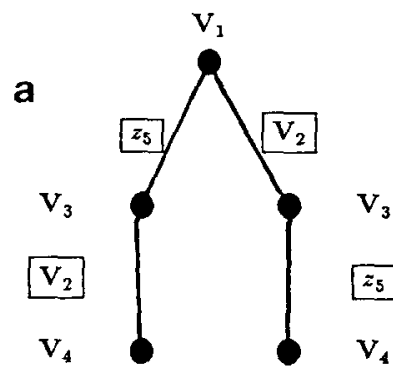

$\mathrm{G}_{2}^{1}(5)$

$\mathrm{G}_{2}^{2}(5)$

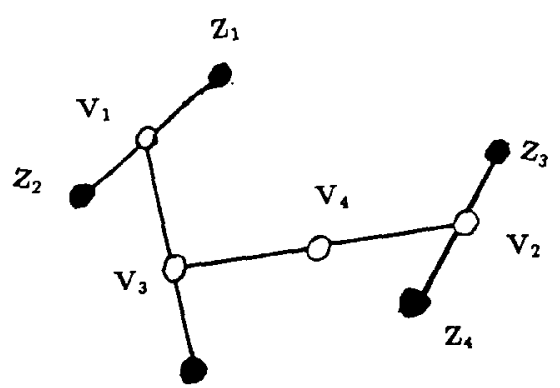

$\mathbf{Z}_{5}$

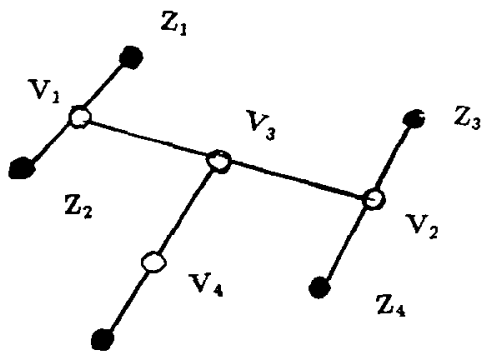

$Z_{5}$
$\mathbf{C}$

Fig. 1. (a) Associated tree, $A_{5}^{2}$; (b), (c) spanning tree graphs, $G_{2}^{s}(5), s=1,2$. 
$\mathrm{V}_{3}=\operatorname{INTER}\left(\mathrm{V}_{1}, \mathrm{~V}_{2}\right)$,

and on the left,

$\mathrm{V}_{3}=\operatorname{INTER}\left(\mathrm{V}_{1}, Z_{5}\right)$.

This is depicted in the trees, $\mathrm{G}_{2}^{s}(s)$, where $s=1,2$ for the left and right branches respectively (cf. figs. 1b, 1c). The next vertex in each branch of $A_{5}^{2}$ is the tier- 3 virtual vertex, $V_{4}$ on the right,

$\mathrm{V}_{4}=\operatorname{INTER}\left(\mathrm{V}_{3}, Z_{5}\right)$

and on the left,

$\mathrm{V}_{4}=\operatorname{INTER}\left(\mathrm{V}_{3}, \mathrm{~V}_{2}\right)$.

There is now no additional branching in $A_{5}^{2}$ and all 4 virtual vertices in $G_{k}^{s}(5), s=1,2$, have been generated. Clearly, all the tier- 0 vertices, $Z_{q}, q=1, \ldots, 5$ and the tier- 1 vertices, $\mathrm{V}_{j}, j=1,2$ have been used only once, thereby satisfying the rules that govern INTER.

The two branches of $A_{5}^{2}$ have the following path representations,

$\mathrm{G}_{2}^{1}(5)=\left\{Z_{5}, \mathrm{v}_{2}\right\}, \quad \mathrm{G}_{2}^{2}(5)=\left\{\mathrm{v}_{2}, Z_{5}\right\}$,

where the boxed quantities denote the edges of $A_{s}^{2}$ to distinguish them from the vertices of $G_{2}^{s}(5)$, after which they were named.

The index $s$ in the label $\mathrm{G}_{k}^{s}(N)$ takes the value of the binary number obtained when the path representation (17) is put in binary form,

$\mathrm{G}_{2}^{1}(5)=\left\{\begin{array}{ll}0 & 1\end{array}\right\}, \mathrm{G}_{2}^{2}(5)=\left\{\begin{array}{ll}1 & 0\end{array}\right\}$,

where 0 denotes a tier- 0 vertex, and a 1 denotes a tier- 1 vertex. This completes the example $A_{S}^{2}$ and gives a description of parts (a) and (b) of our procedure.

Next, we give the third part (c) of our procedure in which the spanning trees $G_{k}^{s}(N)$ are associated with symplectic matrices, $\mathbf{M}_{1}$ and $\mathbf{M}_{2}$ (5), (6). We begin with the rules for $\mathbf{M}_{1}$ and then modify them for $\mathbf{M}_{2}$.

Let

ARG1 $(q)=1$ st argument in INTER that generated $\mathrm{V}_{q}$, the $q$ th virtual vertex,

$\operatorname{ARG} 2(q)=2$ nd argument in the same operation .

In other words,

$\mathrm{V}_{q}=\operatorname{INTER}(\operatorname{ARG} 1(q), \operatorname{ARG} 2(q))$,

and the "weight" of $\mathrm{V}_{q}$ is

$\Gamma\left(\mathrm{V}_{q}\right)=\Gamma(\operatorname{ARG} 1(q))+\Gamma(\operatorname{ARG} 2(q))$.

We remind the reader that the arguments of INTER are complex numbers that give the position of particles or equivalently, vertices in either $\mathbb{R}^{2}$ or $\mathbb{R}^{3}$; the "weights" are real numbers defined by (14).

In the case of $\mathbf{M}_{1}$, for every virtual vertex $\mathrm{V}_{q}$ in $\mathrm{G}_{k}^{s}(N)$, we define

$\rho_{q}=\left[\frac{\Gamma(\operatorname{ARG} 1(q)) \cdot \Gamma(\operatorname{ARG} 2(q))}{\Gamma\left(\mathrm{V}_{q}\right)}\right]^{1 / 2}[\operatorname{ARG} 2(q)-\operatorname{ARG} 1(q)]$ 
for $q=1, \ldots, N-1$ and

$\rho_{N}=\frac{\sum_{j=1}^{N} \Gamma_{j} \mathrm{Z}_{j}}{\sum_{j=1}^{N} \Gamma_{j}}$

Since the arguments of INTER are ultimately linear combinations of the tier-0 vertices, $Z_{j}, j=1, \ldots, N$, formulae (22) define a real linear transformation on $\mathbb{C}^{N}$. Recalling that the symplectic coordinates for first-order Hamiltonians (9) are given by the complex number (cf. (10))

$\omega_{j}=\sqrt{\left|\Gamma_{j}\right|} Z_{j}, \quad j=1, \ldots, N$,

we obtain the desired transformation on $\mathbb{C}^{N}$ by substituting $\omega_{j} / \sqrt{\left|\Gamma_{j}\right|}$ for $Z_{j}$ in $(22 \mathrm{a}, \mathrm{b})$. The matrix of this transformation is the $N \times N$ real constant matrix $\mathbf{A}$ and $\mathbf{M}_{1}$ is given by

$\mathbf{M}_{1}=\left[\begin{array}{ll}\mathbf{A} & 0 \\ 0 & \mathbf{A}\end{array}\right]$.

$\mathbf{M}_{1}$, a $2 N \times 2 N$ real matrix, can be viewed as a transformation from the $2 N$ symplectic coordinates, (10), or the real and imaginary parts of (23) to the $2 N$ coordinates obtained by taking real and imaginary parts of $\rho_{q}$, $q=1, \ldots, N$.

The main result for $\mathbf{M}_{1}$ is

Theorem 1. For any $N$ and $k \leqslant[N / 2]$, the spanning trees $\mathrm{G}_{k}^{s}(N)$ generated by INTER via $\mathrm{A}_{N}^{k}$ define linear canonical transformations (symplectic matrices $\mathbf{M}_{1}$ ) which are explicitly given by (22) and (23).

The proof of this theorem is based on verifying that the matrix $\mathbf{A}$ is orthogonal for each $\mathrm{G}_{k}^{r}(N)$. The graphtheoretic details are given in ref. [8].

In the case of $\mathbf{M}_{2}$, the definitions (19) are valid and the "weights" $\Gamma_{j}$ become masses of the particles, $m_{j}$. We now modify (22) to take into account the asymmetry between the canonical coordinates and momenta of the second-order Hamiltonians (12). For each virtual vertex, $\mathrm{V}_{j}$ in $\mathrm{G}_{k}^{s}(N)$, we write

$Q_{j}=\operatorname{ARG} 2(j)-\operatorname{ARG} 1(j)$,

$P_{j}=\left[\frac{\Gamma(\mathrm{ARG} 1(j)) \cdot \Gamma(\mathrm{ARG} 2(j))}{\Gamma\left(\mathrm{V}_{j}\right)}\right][\mathrm{ARG} 2(j)-\mathrm{AR} G 1(j)]$,

where ARG1 $(j), \operatorname{ARG} 2(j), Q_{j}, P_{j}$ are vectors in $\mathbb{R}^{3}, \Gamma()$ are the masses and ARG $(j)$ denotes the time derivative. This is completed by

$Q_{N}=\frac{\sum_{j=1}^{N} \underline{m_{1}} q_{j}}{\sum_{j=1}^{N} m_{j}}, \quad P_{N}=\frac{\sum_{j=1}^{N} p_{j}}{\sum_{j=1}^{N} m_{j}}$.

The arguments $\dot{A} \dot{R} G 1(j), \operatorname{ARG} 2(j)$ are linear combinations of the tier-0 vertices, $q_{j} \in \mathbb{R}^{3}, j=1, \ldots, N$ while the time derivatives $\dot{A R G}_{1}(j), \mathrm{ARG} 2(j)$ can be written as linear combinations of the conjugate momenta by the substitution

$\frac{p_{j}}{m_{j}}=\dot{q}_{j}$

Therefore, (24) give a linear (real) transformation from $\left(q_{j}, p_{j}\right) \in \mathbb{R}^{3 N} \times \mathbb{R}^{3 N}$ to new coordinates $\left(Q_{j}, P_{j}\right) \in$ 
$\mathbb{R}^{3 N} \times \mathbb{R}^{3 N}$. In fact, (24a) defines the $3 N \times 3 N$ real constant matrix $A$ and after using (25), the second part (24b) defines the $3 N \times 3 N$ matrix, $\mathbf{D}$ in $\mathbf{M}_{2}(6)$.

By (8) the proof [8] of the following theorem is based on checking that $A^{\prime} D=I_{3 N}$ for every $G_{k}^{s}(N)$ generated by the INTER via $A_{N}^{k}$ from the complete graph $K_{N}$ (which in this case is located in $\mathbb{R}^{3}$ ). As far as graph-theory is concerned, the fact that $K_{N}$ in this case is in $\mathbb{R}^{3}$, does not make any difference. The main result for $\mathbf{M}_{2}$ is

Theorem 2. For any $N$ and $k \leqslant[N / 2]$, the spanning trees $\mathrm{G}_{k}^{s}(N)$ define linear canonical transformations (symplectic matrices $\mathbf{M}_{2}$ ) which are explicitly given by $(24 \mathrm{a}, \mathrm{b})$ and $(25)$.

\section{Examples and conclusion}

We present two simple examples of canonical transformations generated by our procedure. One of the examples is a symplectic matrix of type one while the other is of type two but they are both generated from the same spanning tree, $G_{2}^{1}(5)$ (cf. fig. $1 \mathrm{~b}$ ).

Following the procedure discussed in section $3(22)$, for every virtual vertex in $G_{2}^{1}(5)$ there belongs a row in the matrix $A$, given by the complex formulas

$$
\begin{aligned}
& \rho_{1}=\left[\frac{\Gamma_{1} \Gamma_{2}}{\Gamma_{1}+\Gamma_{2}}\right]^{1 / 2}\left(\frac{\omega_{2}}{\sqrt{\Gamma_{2}}}-\frac{\omega_{1}}{\sqrt{\Gamma_{1}}}\right), \quad \rho_{2}=\left[\frac{\Gamma_{3} \Gamma_{4}}{\Gamma_{3}+\Gamma_{4}}\right]^{1 / 2}\left(\frac{\omega_{4}}{\sqrt{\Gamma_{4}}}-\frac{\omega_{2}}{\sqrt{\Gamma_{3}}}\right), \\
& \rho_{3}=\left[\frac{\left(\Gamma_{1}+\Gamma_{2}\right)\left(\Gamma_{5}\right)}{\Gamma_{1}+\Gamma_{2}+\Gamma_{5}}\right]^{1 / 2}\left(\frac{\omega_{5}}{\sqrt{\Gamma_{5}}}-\frac{\sqrt{\Gamma_{1}} \omega_{1}+\sqrt{\Gamma_{2}} \omega_{2}}{\Gamma_{1}+\Gamma_{2}}\right) \text {, } \\
& \rho_{4}=\left[\frac{\left(\Gamma_{1}+\Gamma_{2}+\Gamma_{5}\right)\left(\Gamma_{3}+\Gamma_{4}\right)}{\Gamma_{1}+\Gamma_{2}+\Gamma_{3}+\Gamma_{4}+\Gamma_{5}}\right]^{1 / 2}\left(\frac{\sqrt{\Gamma_{3}} \omega_{3}+\sqrt{\Gamma_{4}} \omega_{4}}{\Gamma_{3}+\Gamma_{4}}-\frac{\sqrt{\Gamma_{1}} \underline{\omega}_{1}+\sqrt{\Gamma_{2}}}{\Gamma_{1}+\Gamma_{2}+\Gamma_{5}+\sqrt{\Gamma_{5}}} \frac{\omega_{5}}{\omega_{5}}\right), \\
& \rho_{5}=\frac{\sum_{i=1}^{5} \sqrt{\Gamma_{i}} \omega_{i}}{\sum_{i=1}^{5} \Gamma_{i}}
\end{aligned}
$$

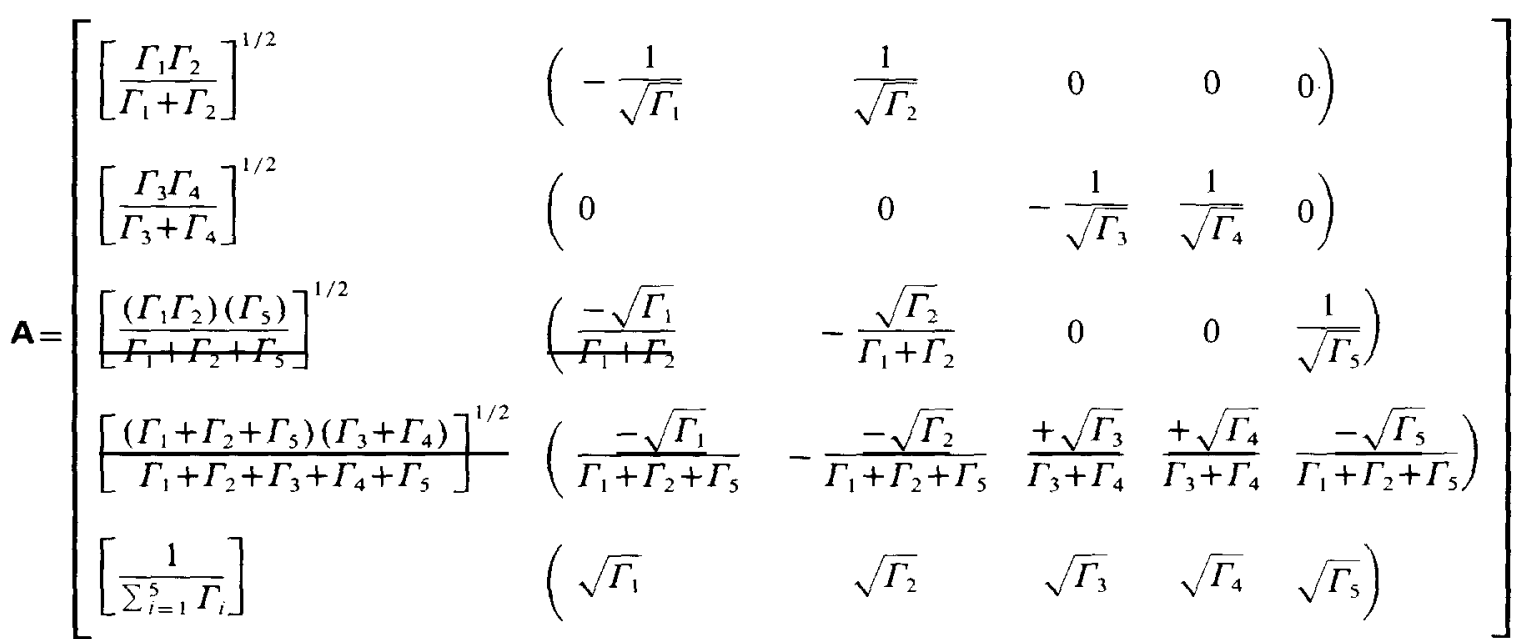

is easily shown to be orthogonal by verifying that the rows form an orthonormal basis for $\mathbb{R}^{5}$. Therefore, the matrix $\mathbf{M}_{1}$ constructed according to (5) is symplectic by ( 7 ).

The above linear transformation together with infinitely many others generated by the same procedure are guaranteed to be canonical transformations by theorem 1. Due to the symmetrical form (5) of these symplectic 
matrices (where $\mathbf{A}$ appears as diagonal blocks), the most natural Hamiltonians to which these canonical transformations apply are the first-order ones, (9). The symmetry between the "coordinates" $q_{j}$ and the "moments" $p_{j}$ is clear since they are essentially the real and imaginary parts of the positions of the particles in the complex plane, (10). In other words, for (9), phase-space can be identified with configuration space. This symmetry sets the first-order problems apart from the second-order Hamiltonians (11), where phase-space $\mathbb{R}^{3 N} \times \mathbb{R}^{3 N}$ can no longer be identified with configuration space $\mathbb{R}^{3 N}$. The second-order problems (11) thus provide the natural setting for applying the canonical transformations associated with symplectic matrices of type two of which an example is given next.

This example of $M_{2}$ is generated by applying (24) to each virtual vertex in the graph $G_{2}^{1}(5)$. The upper left block $A$ is given by $(24 a, c)$,

$\mathbf{A}=\left[\begin{array}{ccccc}-\mathbf{1} & \mathbf{1} & 0 & 0 & 0 \\ \mathbf{0} & 0 & -1 & 1 & 0 \\ -\frac{\mathbf{m}_{1}}{\mathbf{m}_{1}+\mathbf{m}_{2}} & -\frac{\mathbf{m}_{2}}{\mathbf{m}_{1}+\mathbf{m}_{2}} & 0 & 0 & 1 \\ \frac{-\mathbf{m}_{1}}{\mathbf{m}_{1}+\mathbf{m}_{\mathbf{2}}+\mathbf{m}_{5}} & \frac{-\mathbf{m}_{2}}{\mathbf{m}_{1}+\mathbf{m}_{\mathbf{2}}+\mathbf{m}_{5}} & +\frac{\mathbf{m}_{3}}{\mathbf{m}_{3}+\mathbf{m}_{4}} & \frac{+\mathbf{m}_{4}}{\mathbf{m}_{3}+\mathbf{m}_{4}} & \frac{-\mathbf{m}_{5}}{\mathbf{m}_{1}+\mathbf{m}_{2}+\mathbf{m}_{5}} \\ \frac{\mathbf{m}_{1}}{\sum_{j=1}^{5} \mathbf{m}_{j}} & \frac{\mathbf{m}_{2}}{\sum \mathbf{m}_{j}} & \frac{\mathbf{m}_{3}}{\sum \mathbf{m}_{j}} & \frac{\mathbf{m}_{4}}{\sum \mathbf{m}_{j}} & \frac{\mathbf{m}_{5}}{\sum \mathbf{m}_{j}}\end{array}\right]$.

The lower block $\mathrm{D}$ transforms the old momenta, $p_{j}$, into the new momenta, $P_{j}$, and is given by (24b,c); each (bold) entry in $\mathbf{A}$ above and $\mathbf{D}$ below represents a $3 \times 3$ diagonal matrix, for example,

$\mathbf{- 1} \equiv\left[\begin{array}{ccc}-1 & 0 & 0 \\ 0 & -1 & 0 \\ 0 & 0 & -1\end{array}\right], \quad \frac{1}{\mathbf{m}_{2}} \equiv\left[\begin{array}{ccc}m_{2}^{-1} & 0 & 0 \\ 0 & m_{2}^{-1} & 0 \\ 0 & 0 & m_{2}^{-1}\end{array}\right]$.

The $3 N \times 3 N$ real constant matrix $\mathrm{D}$ takes the form

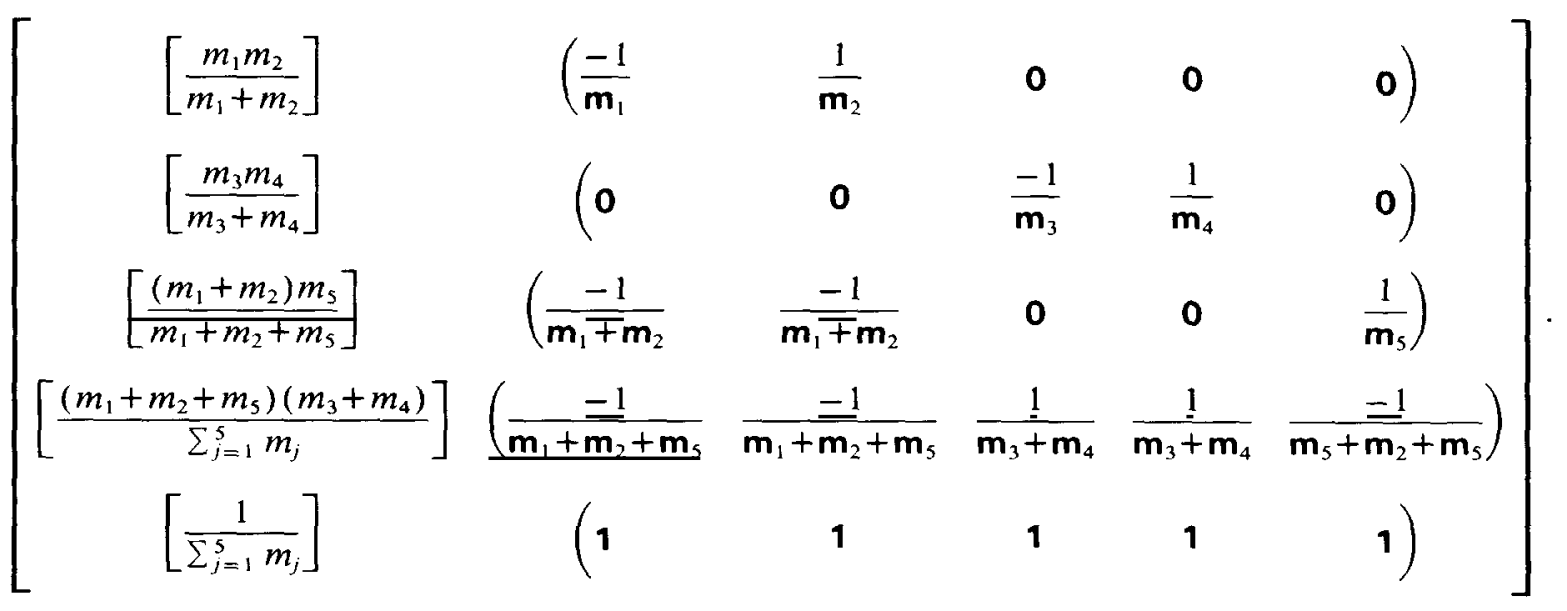

The above symplectic matrix (28), (29) defines a linear canonical transformation for 5-body problems in 
celestial mechanics where it should be noted that the potentials $U(q)(12)$ are not restricted to the gravitational square force-law (cf. ref. [5]). In fact, it gives an example of the so-called Jacobi coordinates, well-known in the 3-body problem [5]. Theorem 2 can be viewed as stating that in the class of $3 N \times 3 N$ matrices of the form (6) there exists an infinite subset that is generated by binary trees $G_{k}^{s}(N)$, and which satisfies the condition for being symplectic, (8). These symplectic matrices, $\mathbf{M}_{2}$, give a sweeping generalization of the Jacobi coordinates, which are discussed only in special cases (for example the 3-body problem) in the literature.

The canonical transformations defined by $\mathbf{M}_{1}$ are originally conceived to study clustering phenomena in vortex dynamics $[12,13]$. Clearly, the range of applications for these transformations is eonsiderably wider, in fact no further restrictions on the form of the functions $F_{j k}$ in (9) are necessary for these transformations to work. Similarly, the Jacobi coordinates defined by $\mathbf{M}_{2}$ can be applied to the study of clusters in stellar dynamics [14]. In fact, the so-called cluster coordinates discussed in McGehee's paper [6] are known to be symplectic coordinates $\$ 2$. This provides the point of departure for an extension of the ideas presented to symplectic matrices generated by spanning trees more general than binary ones.

\section{Acknowledgement}

The author has benefited from discussions with R. McGehee, School of Mathematics, University of Minnesota, as well as numerous other people including members of the IMA: D.D. Holm, D. Kaup, Y. Kodama, P. Olver and D. Sattinger. The author would like to acknowledge the support of the IMA where part of this research was done. He would like to thank Patricia V. Brick for her expert and prompt typing of this manuscript.

\#2 Richard McGehee, School of Mathematics, University of Minnesota communicated this to the author.

\section{References}

[1] N. Nakanishi, Graph theory and Feynman integrals (Gordon and Breach, New York, 1970).

[2] G. Uhlenbeck and G. Ford, Lectures in statistical mechanics (Gordon and Breach, New York, 1960).

[3] H. Lamb, Hydrodynamics (Dover, New York).

[4] D. Montgomery and G. Joyce, Phys. Fluids 17 (1974) 1139.

[5] H. Pollard, A mathematical introduction to celestial mechanics, MAA monograph.

[6] R. McGehee, Exp. Math. 4 (1986) 335.

[7] D. Saari, Arch. Rat. Mechanics Anal. 49 (1973) 311.

[8] Ch.C. Lim, On symplectic tree graphs, IMA Preprint Series 465 (1988), submitted for publication.

[9] Ch.C. Lim, Spanning binary trees, symplectic matrices and canonical transformations for classical $N$-body problems, IMA Preprint Series \#475, submitted for publication.

[10] N.L. Biggs, Algebraic graph theory (Cambridge Univ. Press, Cambridge, 1975).

[11] R.J. Wilson, Introduction to graph theory (Oliver and Boyd, Edinburgh, 1972).

[12] Ch.C. Lim, Physica D 30 (1988) 343.

[13] Ch.C. Lim, On singular Hamiltonians: existence of quasi-periodic solutions and nonlinear stability, AMS Bull. (January 1989).

[14] S. Chandrasekhar, Principles of stellar dynamics (Univ. of Chicago Press, Chicago, 1942). 